\title{
VALIDADE DO NÍVEL DE ESCOLARIDADE E INCLUSÃO NO PROCESSO PRODUTIVO POR MEIO DE ANÁLISE DE CORRELAÇÃO
}

\author{
Alvaro Martim GUEDES ${ }^{1}$ \\ Camila Fernanda BASSETTO ${ }^{2}$
}

Resumo: O presente artigo visa demonstrar, por meio de base empírica e análise de correlação, a validade da afirmação de que a inclusão no mercado de trabalho está diretamente correlacionada com o grau de instrução, ou seja, com a formação obtida pelo indivíduo. As fontes de dados foram o Instituto Brasileiro de Geografia e Estatística, IBGE, e o de Setores de Atividade Econômica da Relação Anual de Informações Sociais, RAIS. A análise realizada abrangeu o período de 2002 a $2012 \mathrm{em}$ um Município do Estado de São Paulo, com população de aproximadamente $220 \mathrm{mil}$ habitantes. $\mathrm{O}$ mérito do estudo realizado foi o de demonstrar com base empírica e meios de análise consagrados de estatística que de fato há evidente correlação entre formação escolar, colocações no mercado de trabalho dos indivíduos e desenvolvimento econômico. Essa observação foi obtida com base no nível salarial, gênero e setores econômicos (primário, secundário e terciário). Ademais, foi observado a correlação existente entre desenvolvimento econômico e formação escolar, o que fortalece a afirmação generalizada da função estratégica da Educação para um país.

Palavras-chave: Desenvolvimento regional. Análise de correlação. Nível de escolaridade.

\section{INTRODUÇÃO}

O presente artigo deriva de estudos e pesquisas realizados para a compreensão e validade da análise de correlação (técnica estatística), dados indicadores de emprego, renda e formação de trabalhadores. O conteúdo aqui exposto foi apresentado em outro evento, contudo, a ênfase dada foi quanto à validade da análise. Neste artigo é dada ênfase à validade da escolaridade na inserção da mão de obra no mercado de trabalho e à correlação desta com o processo de desenvolvimento regional.

As fontes de dados (IBGE e RAIS) e a circunscrição da base empírica à somente uma cidade ocorreram em função de vir a comprovar, ou não, a correlação entre escolaridade e desenvolvimento econômico sem que outas possíveis variáveis afetassem

\footnotetext{
${ }^{1}$ Departamento de Administração Pública. UNESP - Universidade Estadual Paulista Júlio de Mesquita Filho. Faculdade de Ciências e Letras. Araraquara - SP - Brasil. 14800-901 - alvaro@fclar.unesp.br.

2 Departamento de Ciências da Educação. UNESP - Universidade Estadual Paulista Júlio de Mesquita Filho. Faculdade de Ciências e Letras. Araraquara - SP - Brasil. 14800-901 - camilafclar@gmail.com.
} 
de forma significativa o estudo realizado. Isso porque, ao circunscrever a somente uma localidade, que é capaz com certa autonomia formar indivíduos, dada a sua estrutura educacional, fatores outros, como migração, por exemplo, puderam não ser cotejados com segurança.

Ao final, o estudo possibilitou comprovar a existência dessa correlação, entre escolarização e desenvolvimento econômico, o que muito auxilia na elaboração de ações de estado com vistas a promover a educação formal.

\section{Contextualização da evolução da força de trabalho no Brasil nas últimas décadas}

O mercado de trabalho no Brasil tem apresentado relevantes alterações. A qualificação da mão-de-obra foi uma dessas modificações ao apresentar uma elevação. Isso ocorreu dado o aumento na escolaridade dos trabalhadores brasileiros. Esse novo cenário de transformações também é possível de ser comprovado dada a evolução dos salários. Esses fatores, segundo Fernandes e Menezes-Filho (2002), podem ser verificados no aumento crescente, nas últimas décadas, da demanda por trabalhadores mais qualificados em tarefas consideradas mais complexas. Para Menezes-Filho e Rodrigues Júnior (2003), um dos principais fatores que contribuem para a compreensão do aumento da demanda por mão-de-obra mais qualificada no Brasil, é a transferência de tecnologia, o que exige maior escolaridade dos trabalhadores. Por outro lado, De Pauli, Nakabashi e Sampaio (2012) mostraram que, embora os ganhos de produtividade na indústria de transformação e no setor de serviços não tenha aumentado a demanda por trabalhadores qualificados, as quantidades de trabalhadores com qualificação intermediária, isto é, níveis de ensino Fundamental e Médio completos aumentaram expressivamente. Os autores destacaram ainda que "[...] aumentos da escolaridade média dos trabalhadores brasileiros responderam, em maior medida, à expansão da oferta de ensino no país." (DE PAULI; NAKABASHI; SAMPAIO, 2012, p.461). Sendo assim, a partir de 2004, os indicadores do mercado de trabalho relacionados ao grau de escolaridade começaram a registrar melhoras significativas.

De acordo com Campos (2003), já na década de 90, o Brasil apresentou uma melhora significativa nos seus índices educacionais quantitativos, reduziu a taxa de analfabetismo e aumentou o nível médio de escolaridade da população, ainda que a um ritmo inferior quando comparado a décadas anteriores. Concomitantemente, porém, o mercado de trabalho naquela década foi marcado por uma forte crise com altas taxas de desemprego de longa duração, aumento da informalidade, retração do emprego formal, 
deterioração dos níveis de renda, especialmente entre as faixas etárias mais jovens. Essa degradação do mercado de trabalho persistiu ao longo dos anos de 1990. Borges (2010) argumentou que, acompanhada das incertezas relacionadas ao vínculo empregatício, incluindo desemprego, perdas salariais e benefícios, essa crise afetou diversos segmentos, incluindo aqueles considerados mais protegidos, compostos por servidores públicos e empregados das grandes empresas públicas e privadas, e também os trabalhadores dos segmentos mais reestruturados, como os operários da moderna indústria e aqueles que integram a chamada força de trabalho secundária, abrangendo jovens e mulheres. Nesse cenário desfavorável os que arcaram maiores ônus foram aqueles com baixa escolaridade.

Na primeira década do século XXI, mais especificamente a partir de 2004, como resultado da confluência de fatores internos e externos favoráveis, o país vivenciou uma aceleração no processo produtivo. A crise global que se seguiu a esse período, ocorrida no último trimestre de 2008, embora tenha atuado negativamente sobre a economia brasileira, não impediu a expansão do consumo e da produção, o que refletiu positivamente sobre a capacidade de geração de novos postos de trabalho. Paralelamente a essa fase, importantes mudanças sociodemográficas atuaram sobre a estrutura e a dinâmica do mercado de trabalho. Uma dessas mudanças é caracterizada pela aceleração no processo de entrada das mulheres no mercado de trabalho. A elevação do nível de escolaridade dos ofertantes de trabalho, beneficiados pela expansão do sistema educacional nas duas últimas décadas (1990 a 2010), também exerceu impacto sobre o mercado de trabalho. Articuladas, essas mudanças resultaram em diferentes condições para a inserção de homens, mulheres, jovens, adultos e idosos no mercado de trabalho, em conformidade com o nível de escolaridade.

\section{Aspectos metodológicos e contextualização do Município escolhido}

O presente estudo tem como propósito fornecer uma metodologia de análise empírica baseada nas correlações existentes entre o nível de escolaridade e o desenvolvimento econômico de uma cidade de porte médio do estado de São Paulo. Para alcançar tal objetivo, foram considerados dados referentes ao período compreendido entre os anos de 2002 a 2012, coletados no Instituto Brasileiro de Geografia e Estatística - IBGE (2013) e também dos Setores de Atividade Econômica da Relação Anual de Informações Sociais - RAIS (2013). Os dados correspondem ao nível de instrução da população, discriminado por gênero e setores da indústria, ao 
rendimento médio total e por trabalhador, incluindo as variações percentuais no período considerado, ao produto interno bruto, e outras informações.

A cidade caracteriza-se como um polo regional, que atrai consumidores de outras cidades no entorno, possui um relevante comércio atacadista e varejista, indústrias, produção agrícola e empresas prestadoras de serviços.

Os dados do IBGE (2013), entre 2002 e 2012, mostraram que o PIB do município mais que duplicou no período considerado. Enquanto em 2002 o PIB era de $\mathrm{R} \$ 2.091,25$ milhões, em 2012 foi aproximadamente $\mathrm{R} \$$ 5.600,00 milhões. Exceto de 2003 a 2004 a variação percentual do PIB local foi positiva. Dentre tais variações, destaca-se aquela ocorrida entre 2004 e 2005, a qual alcançou o maior valor registrado correspondendo a aproximadamente $20 \%$, logo em seguida ao pior valor observado entre 2003 e 2004, igual a -0,4. Entre 2006 e 2012 o comportamento da variação percentual do PIB foi semelhante, ocorrendo queda seguida de alta.

O nível de escolaridade da população do Município é apresentado na Tabela 1.

Tabela 1 - Grau de instrução da população entre 2002 e 2013

\begin{tabular}{lcccccccccccc}
\hline $\begin{array}{l}\text { Nível } \\
\text { de Inst. }\end{array}$ & $\mathbf{2 0 0 2}$ & $\mathbf{2 0 0 3}$ & $\mathbf{2 0 0 4}$ & $\mathbf{2 0 0 5}$ & $\mathbf{2 0 0 6}$ & $\mathbf{2 0 0 7}$ & $\mathbf{2 0 0 8}$ & $\mathbf{2 0 0 9}$ & $\mathbf{2 0 1 0}$ & $\mathbf{2 0 1 1}$ & $\mathbf{2 0 1 2}$ & $\mathbf{2 0 1 3}$ \\
\hline Analf. & 572 & 328 & 291 & 201 & 222 & 239 & 266 & 353 & 239 & 234 & 173 & 126 \\
\hline $\begin{array}{l}\text { Fun. I } \\
\text { Comp. }\end{array}$ & 7.014 & 6.424 & 6.756 & 8.575 & 7.442 & 5.844 & 4.996 & 5.521 & 4.148 & 4.867 & 3.862 & 3.400 \\
\hline $\begin{array}{l}\text { Fun. II } \\
\text { Comp. }\end{array}$ & 8.526 & 8.629 & 9.306 & 10.172 & 11.063 & 12.049 & 13.415 & 14.024 & 14.097 & 15.433 & 14.533 & 13.059 \\
\hline $\begin{array}{l}\text { Médio } \\
\text { Comp. }\end{array}$ & 11.951 & 13.286 & 14.723 & 16.426 & 18.981 & 21.849 & 23.926 & 25.628 & 27.646 & 32.470 & 33.952 & 35.792 \\
\hline $\begin{array}{l}\text { Sup. } \\
\text { Comp. }\end{array}$ & 4.923 & 5.534 & 5.406 & 5.903 & 6.432 & 6.870 & 6.976 & 7.641 & 8.234 & 9.102 & 9.282 & 9.843 \\
\hline Total & 32.986 & 34.201 & 36.482 & 41.277 & 44.130 & 46.851 & 49.579 & 54.163 & 54.364 & 62.106 & 61.802 & 62.221 \\
\hline
\end{tabular}

Fonte: Elaboração própria a partir de dados coletados na RAIS (2013).

Conforme observado na Tabela 1, o nível de escolaridade dos habitantes aumentou no período analisado. Enquanto em 2002, 572 pessoas caracterizavam-se como analfabetas, em 2013 esse número foi reduzido quase 80\%, atingindo apenas 126 pessoas. Quanto à quantidade de pessoas que cursaram até o Ensino Fundamental I Completo o número observado foi de 7.014 em 2002 caindo para 3.400 em 2013. Destaca-se os anos de 2005 e 2006, onde o número de pessoas com este nível de escolaridade atingiu os valores máximos iguais a 8.575 e 7.442 , respectivamente. A redução no número de pessoas nessa categoria é explicada pelo aumento daqueles que conseguiram completar o Ensino Fundamental II, pois enquanto 8.526 pessoas 
pertenciam a essa categoria em 2002, essa quantidade alcançou 13.059 pessoas em 2013, correspondendo a um aumento de mais de 50\%. Os números de pessoas com o Ensino Médio Completo e com Superior Completo também cresceram substancialmente. Enquanto em 2002 aproximadamente 12 mil pessoas possuíam o Ensino Médio Completo, em 2013 esse número foi quase 36 mil pessoas, isto é, três vezes mais, representando um aumento de $200 \%$. Destaca-se também o número de pessoas com diplomas, uma vez que tal quantidade passou de aproximadamente cinco mil pessoas em 2002 para quase dez mil em 2013.

O nível de escolaridade da população do Município em questão também foi analisado por gênero entre os anos de 2002 e 2013. Os dados são apresentados na Tabela 2.

A Tabela 2 mostra que, entre os homens, o percentual de Analfabetos passou de 1,38 em 2002 para 0,23 em 2013, caracterizando uma redução de $0,83 \%$ no período considerado.

Tabela 2 - Valores percentuais de homens e mulheres com diferentes níveis de escolaridade

\begin{tabular}{lcccccccccccc}
\hline Masc. & $\mathbf{2 0 0 2}$ & $\mathbf{2 0 0 3}$ & $\mathbf{2 0 0 4}$ & $\mathbf{2 0 0 5}$ & $\mathbf{2 0 0 6}$ & $\mathbf{2 0 0 7}$ & $\mathbf{2 0 0 8}$ & $\mathbf{2 0 0 9}$ & $\mathbf{2 0 1 0}$ & $\mathbf{2 0 1 1}$ & $\mathbf{2 0 1 2}$ & $\mathbf{2 0 1 3}$ \\
\hline Analf. & 1,38 & 0,79 & 0,66 & 0,46 & 0,48 & 0,48 & 0,50 & 0,68 & 0,43 & 0,43 & 0,32 & 0,23 \\
\hline $\begin{array}{l}\text { Fun. I } \\
\text { Comp. }\end{array}$ & 17,88 & 16,68 & 16,45 & 20,62 & 16,45 & 12,46 & 10,48 & 10,54 & 7,77 & 8,19 & 6,28 & 5,48 \\
\hline $\begin{array}{l}\text { Fun. II } \\
\text { Comp. }\end{array}$ & 19,91 & 20,80 & 22,10 & 21,29 & 21,64 & 22,92 & 23,97 & 23,07 & 23,45 & 22,26 & 22,24 & 19,77 \\
\hline $\begin{array}{l}\text { Médio } \\
\text { Comp. }\end{array}$ & 20,81 & 23,72 & 25,10 & 25,42 & 28,70 & 31,84 & 33,89 & 35,41 & 36,97 & 37,44 & 41,61 & 44,61 \\
\hline $\begin{array}{l}\text { Sup. } \\
\text { Comp. }\end{array}$ & 7,75 & 9,03 & 8,41 & 8,45 & 8,86 & 8,84 & 8,61 & 8,81 & 9,70 & 9,45 & 10,16 & 11,17 \\
\hline Fem. & $\mathbf{2 0 0 2}$ & $\mathbf{2 0 0 3}$ & $\mathbf{2 0 0 4}$ & $\mathbf{2 0 0 5}$ & $\mathbf{2 0 0 6}$ & $\mathbf{2 0 0 7}$ & $\mathbf{2 0 0 8}$ & $\mathbf{2 0 0 9}$ & $\mathbf{2 0 1 0}$ & $\mathbf{2 0 1 1}$ & $\mathbf{2 0 1 2}$ & $\mathbf{2 0 1 3}$ \\
\hline $\begin{array}{l}\text { Analf. } \\
0,99\end{array}$ & 0,58 & 0,53 & 0,26 & 0,26 & 0,30 & 0,33 & 0,32 & 0,25 & 0,13 & 0,12 & 0,10 \\
\hline $\begin{array}{l}\text { Fun. I } \\
\text { Comp. }\end{array}$ & 10,5 & 9,63 & 10,64 & 9,67 & 8,23 & 5,73 & 4,45 & 5,17 & 4,00 & 3,77 & 3,79 & 3,43 \\
\hline $\begin{array}{l}\text { Fun. II } \\
\text { Comp. }\end{array}$ & 15,95 & 15,59 & 15,53 & 16,65 & 16,88 & 16,45 & 18,34 & 18,76 & 17,76 & 17,18 & 16,01 & 14,66 \\
\hline $\begin{array}{l}\text { Médio } \\
\text { Comp. }\end{array}$ & 34,73 & 37,76 & 40,07 & 41,55 & 42,90 & 46,10 & 46,29 & 44,48 & 47,40 & 49,30 & 50,32 & 51,41 \\
\hline $\begin{array}{l}\text { Sup. } \\
\text { Comp. }\end{array}$ & 15,75 & 17,14 & 15,98 & 16,06 & 15,97 & 16,46 & 15,44 & 15,90 & 15,95 & 15,28 & 15,28 & 15,42 \\
\hline
\end{tabular}

Fonte: Elaboração própria a partir de dados coletados na RAIS (2013). 
Para as mulheres, enquanto o percentual de Analfabetas em 2002 era de 0,99, em 2013 esse número foi significativamente menor, igual a 0,10, evidenciando uma queda de $90 \%$. Nota-se também que, enquanto o percentual de mulheres com nível de instrução até o Ensino Fundamental I foi decrescente para a maioria dos anos, para os homens, embora tenha havido queda, em 2005 esse percentual aumentou mais de 4\% comparado ao ano anterior e, em 2011, atingiu 8,19\%. Para ambos os gêneros, o percentual com o Ensino Médio Completo foi crescente entre 2002 e 2013 e com o ensino Superior Completo, o comportamento dos percentuais foram semelhantes entre os dois grupos, ora crescente, ora decrescente. Comportamento semelhante é apresentado pelos percentuais de mulheres com ensino Superior Completo, isto é, o percentual de mulheres com diploma de graduação é superior ao percentual de homens com tal nível de escolaridade.

A Tabela 3 contém valores referentes à Remuneração Média Total, Total de Vínculo Ativo e Remuneração Média por Trabalhador na cidade de Araraquara, entre 2002 e 2013.

Tabela 3 - Remuneração Média Total e por Trabalhador, em R\$, e Total de Vínculo Ativo

\begin{tabular}{cccc}
\hline Ano & $\begin{array}{c}\text { Remuneração Média } \\
\text { Total (em R\$) }\end{array}$ & $\begin{array}{c}\text { Total de Vínculo } \\
\text { Ativo }\end{array}$ & $\begin{array}{c}\text { Remuneração Média por } \\
\text { Trabalhador (em R\$) }\end{array}$ \\
\hline 2002 & $36.516 .820,00$ & 46.171 & 790,90 \\
2003 & $40.539 .353,54$ & 45.809 & 884,96 \\
2004 & $44.152 .903,32$ & 47.608 & 927,43 \\
2005 & $51.621 .571,16$ & 52.081 & 991,18 \\
2006 & $61.147 .106,09$ & 55.786 & $1.096,10$ \\
2007 & $68.601 .172,49$ & 58.822 & $1.166,25$ \\
2008 & $78.136 .026,25$ & 62.023 & $1.259,79$ \\
2009 & $89.032 .123,11$ & 66.035 & $1.348,26$ \\
2010 & $98.179 .297,39$ & 67.259 & $1.459,72$ \\
2011 & $119.562 .107,08$ & 76.983 & $1.553,10$ \\
2012 & $141.885 .487,02$ & 75.373 & $1.882,44$ \\
2013 & $129.065 .879,53$ & 74.989 & $1.721,13$ \\
\hline Total & $\mathbf{9 5 8 . 4 3 9 . 8 4 6 , 9 8}$ & $\mathbf{7 2 8 . 9 3 9}$ & $\mathbf{1 5 . 0 8 1 , 2 6}$ \\
\hline
\end{tabular}

Fonte: Elaboração própria a partir de dados coletados na RAIS (2013).

De acordo com a Tabela 3, a Remuneração Média Total foi crescente em todo o período considerado, sendo em 2011 quase três vezes mais alta quando comparada com o ano de 2003. Pode-se observar também que a maior taxa percentual de crescimento 
ocorrida foi de aproximadamente 22\% entre os anos de 2011 e 2012, uma vez que a Remuneração Média Total passou de R\$ 119.562.107,08 em 2011 para R\$ 141.885.487,02 em 2012. A Tabela 3 contém ainda valores referentes ao Total de Vínculo Ativo e à Remuneração Média por Trabalhador entre 2003 e 2013. É possível notar que em 2010 ocorreu a maior taxa percentual de crescimento no Total de Vínculo Ativo, aproximando-se dos 15\%, passando de 67.259 em 2010 para 76.983 em 2011, representando um aumento de quase 10 mil novos vínculos ativos na cidade. Por outro lado, entre 2009 e 2010 foi registrado o menor crescimento percentual no Total de Vínculo Ativo. Enquanto em 2009, o total era de 66.035, em 2010 passou a ser de 67.259, correspondendo a um aumento de menos de $2 \%$. No período considerado, a Remuneração Média por Trabalhador foi crescente, alcançando valor máximo em 2012.

\section{Análise de correlação entre desenvolvimento econômico e nível de escolaridade}

De acordo com a visão de capital humano (MINCER, 1958), infere-se que, quanto maior o nível de escolaridade de um indivíduo maior sua produtividade o que acarretará elevação do crescimento econômico.

Na literatura brasileira sobre a relação entre desenvolvimento econômico e nível educacional, destacam-se os estudos de Dias J., Dias M. e Lima (2005), Nakabashi e Figueiredo (2008), Viana e Lima (2010), entre outros. Utilizando um banco de dados com informações sobre os estados brasileiros, Dias J., Dias M. e Lima (2005) desenvolveram um modelo teórico pressupondo o capital humano como causa do crescimento econômico. Os resultados mostraram que aumentos nos níveis de escolaridade geram efeitos nas taxas de crescimento do PIB per capita, evidenciando a importância das políticas de acumulação de capital humano para o aumento do PIB.

Nakabashi e Figueiredo (2008) analisaram os efeitos diretos e indiretos do capital humano sobre a taxa de crescimento da renda e concluíram que "[...] o papel do capital humano sobre a taxa de crescimento econômico é por meio do seu estímulo à difusão de tecnologia. Seus impactos diretos na melhora das habilidades dos trabalhadores e criação de tecnologia não são relevantes". (NAKABASHI; FIGUEIREDO, 2008, p.165).

No estudo de Viana e Lima (2010), o objetivo foi analisar a influência do grau de escolaridade do indivíduo, citada como capital humano, no crescimento econômico e também a possibilidade de utilizá-lo como alternativa de desconcentração desse crescimento. Para os autores, “[...] a ideia de investir em capital humano parte do 
princípio que uma força de trabalho instruída, com qualidade de vida, com acesso aos serviços sociais básicos e plena cidadania será mais produtiva e eficaz nas suas atividades". (VIANA; LIMA, 2010, p.146).

A análise aqui realizada constitui-se da verificação da existência de correlação ${ }^{3}$ entre PIB, Renda Média por Trabalhador (RMT), Total de Vínculo Ativo (TVA) e níveis de escolaridade para os indivíduos da cidade considerada.

Na Tabela 4 são apresentados os valores obtidos para a correlação entre o PIB, a RMT e o TVA, registrados no município em questão. Como esperado, as variáveis citadas são altamente correlacionadas, uma vez que apresentaram valores próximos de um, evidenciando a existência de uma relação positiva entre os valores do PIB, RMT e TVA. Conclui-se o PIB da cidade aumenta à medida que a RMT e o TVA aumentam, e sofrerá queda caso contrário. O mesmo vale tomando-se como referencia a RMT ou o TVA.

Tabela 4 - Correlações entre PIB, Renda Média por Trabalhador e Total de Vínculo Ativo

\begin{tabular}{cccc}
\hline & PIB & RMT & TVA \\
\hline PIB & 1,000 & 0,993 & 0,982 \\
RMT & 0,992 & 1,000 & 0,989 \\
TVA & 0,982 & 0,989 & 1,000 \\
\hline
\end{tabular}

Fonte: Elaboração própria.

Na verificação da existência de uma relação entre o desenvolvimento econômico da cidade com o nível de escolaridade, as correlações entre PIB, RMT e TVA com os diferentes níveis de escolaridade registrados foram calculadas. Os valores obtidos para as correlações entre tais variáveis são mostrados na Tabela 5.

${ }^{3}$ A correlação, representada por $\rho=\frac{n \sum x y-\left(\sum x\right)\left(\sum y\right)}{\sqrt{n \sum x^{2}-\left(\sum x\right)^{2}} \sqrt{n \sum y^{2}-\left(\sum y\right)^{2}}}$, onde $n$ é o número de pares de dados, é uma medida da força e direção de uma relação linear entre duas variáveis. A amplitude da correlação é -1 para um. Enquanto valores próximos de -1 caracterizam uma relação negativa forte, valores próximos de um mostram uma correlação positiva forte. Valores de $\rho$ próximos a $-0,5$ e 0,5 evidenciam correlação fraca e $\rho \approx 0$ mostram ausência de correlação (LARSON; FARBER, 2010). 
Tabela 5 - Correlação entre PIB, RMT e TVA com o nível de escolaridade

\begin{tabular}{|c|c|c|c|c|c|c|c|c|c|}
\hline & A & $\begin{array}{l}\text { Fund. I } \\
\text { Incomp. }\end{array}$ & $\begin{array}{l}\text { Fund. } \\
\text { I } \\
\text { Comp. }\end{array}$ & $\begin{array}{l}\text { Fund. II } \\
\text { Incomp. }\end{array}$ & $\begin{array}{c}\text { Fund. } \\
\text { II } \\
\text { Comp. }\end{array}$ & $\begin{array}{l}\text { Médio } \\
\text { Incomp. }\end{array}$ & $\begin{array}{l}\text { Médio } \\
\text { Comp. }\end{array}$ & $\begin{array}{c}\text { Sup. } \\
\text { Incomp. }\end{array}$ & $\begin{array}{l}\text { Sup. } \\
\text { Comp. }\end{array}$ \\
\hline PIB & $\stackrel{-}{0.530}$ & -0.462 & -0.835 & 0.216 & 0.958 & 0.884 & 0.991 & 0.979 & 0.773 \\
\hline RMT & $\begin{array}{c}- \\
0.522 \\
\end{array}$ & -0.413 & -0.817 & 0.252 & 0.950 & 0.902 & 0.995 & 0.972 & 0.781 \\
\hline TVA & $\begin{array}{c}- \\
0.510\end{array}$ & -0.428 & -0.781 & 0.276 & 0.979 & 0.936 & 0.993 & 0.990 & 0.766 \\
\hline
\end{tabular}

Fonte: Elaboração própria a partir do cálculo da correlação entre as variáveis.

A Tabela 5 mostra que os maiores valores referem-se à correlação entre as variáveis PIB, RMT e TVA com os níveis de instrução correspondentes ao ensino Médio Comp. (Médio Completo), seguido por Sup. Incomp. (Superior Incompleto), Fund. II Comp. (Fundamental II Completo) e Médio Incomp. (Médio Incompleto). Os valores obtidos para as correlações citadas variam entre 0.884 e 0.995 , evidenciando uma relação positiva muito forte entre tais variáveis. Estes valores próximos de um sugerem que pessoas com tais níveis de instrução auxiliam positiva e significativamente para o aumento do PIB da cidade e também da RMT e TVA. Embora com correlação um pouco mais baixa, o grau de escolaridade correspondente ao ensino Médio Incomp. (Médio Incompleto), também exerce papel significativo sobre tais variáveis econômicas.

Por outro lado, trabalhadores com o ensino Fund. I Comp. (Fundamental I Completo) atuam negativamente, mas em nível moderado, sobre as variáveis consideradas relacionadas ao desenvolvimento econômico do município em estudo. A forte relação negativa existente entre tais variáveis e o nível de escolaridade é explicitada por valores negativos e próximos de 0.8 , sugerindo que, à medida que aumenta o número de pessoas com esse nível de instrução e atuantes nas atividades econômicas da cidade, a tendência é que valores mais baixos para o PIB, a RMT e o TVA diminuam. As quantidades de A (Analfabetos) e pessoas que não concluíram o ensino Fundamental I (Fund. I Incomp) exercem papel negativo sobre o desenvolvimento econômico do município, porém de nível considerado fraco. Com valores de correlação próximos de zero, o nível de instrução caracterizado pelo ensino Fundamental II Incompleto (Fund. II Incomp.) evidencia ausência de relação com as variáveis econômicas. 
As tabelas de correlação mostram que o nível de escolaridade exerce papel significante sobre o crescimento econômico desta cidade em estudo. Os valores obtidos para a correlação entre as variáveis PIB, RMT e TVA e os diferentes níveis de escolaridades dos trabalhadores atuantes na cidade e em setores de atividades específicos explicitam a forte relação positiva existente com os níveis de instrução mais altos, isto é, a partir do Fundamental II Completo. Desse grau de escolaridade para baixo, ou existe uma relação negativa entre tais variáveis ou não há relação.]

\section{Conclusão}

O presente estudo teve como propósito a compreensão e validade da análise de correlação para dados de indicadores de emprego, renda e formação de trabalhadores, com ênfase no nível de instrução para inserção da mão de obra no mercado de trabalho e à correlação desta com o processo de desenvolvimento regional. Foram considerando dados referentes os anos de 2002 e 2013, coletados no IBGE (2013) e na RAIS (2013), referentes ao nível de instrução da população, discriminado por gênero, ao rendimento médio total e por trabalhador, incluindo as variações percentuais no período considerado, ao produto interno bruto entre outras informações.

Os resultados a partir da análise de correlação mostram que há seguras evidências de que a educação é um meio de contribuição eficaz, por preparar trabalhadores para o mercado de trabalho, melhorar seus conhecimentos e habilidade. Tal evidência apoia-se nas correlações obtidas para as variáveis de desenvolvimento econômico consideradas com o nível de escolaridade da população do município em análise. Níveis mais altos de instrução da população contribuem de forma positiva e significativa para o crescimento econômico, enquanto níveis mais baixos de escolaridade, ora comportam-se como vilões do desenvolvimento do município por seguirem na direção oposta ao crescimento, ora são insignificantes.

Todas essas afirmações em seu conjunto permitem concluir que tanto a análise de correlação é factível quanto possibilita conclusões seguras quanto ao nível de escolaridade e, portanto, de qualificação da mão de obra e de sua relação direta com desenvolvimento econômico local. Sendo assim, a técnica utilizada, suas fontes de dados e os impactos econômicos verificados confirmam a hipótese corrente de que é possível fazer tal verificação e de que de fato há essa correlação. Embora aparenta ser uma análise restrita, é demonstrada a possibilidade de obtenção de base empírica para tal tipo de análise quanto ao desenvolvimento econômico local. 


\section{VALIDITY OF LEVEL EDUCATION AND INCLUSION IN PRODUCTION PROCESS BY CORRELATION ANALYSIS}

Abstract: This article seeks to demonstrate through empirical basis and correlation analysis, the validity of the statement that the inclusion in the labor market is directly correlated with the level of education, ie with the training received by the individual. The data sources were the Brazilian Institute of Geography and Statistics, IBGE, and Sectors of Economic Activity of the Annual Social Information, RAIS. The analysis covered the period 2002 to 2012 in a municipality of São Paulo, with a population of approximately 220,000 inhabitants. The study conducted on the merits was to demonstrate empirically based and analytical resources devoted to statistics that in fact there is clear correlation between school education placements in the labor market of individuals and economic development. This observation was obtained based on salary level, gender and economic sectors (primary, secondary and tertiary). Moreover, the correlation was observed between economic development and schooling, which strengthens the widespread assertion of Education strategic role for a country.

Key words: Education and develompent. Regional Economic Development. Correlation analysis.

\section{REFERÊNCIAS}

BORGES, A. As novas configurações do mercado de trabalho urbano no Brasil: notas para discussão. Caderno CRH, Salvador, v.23, n.60, p.619-632, 2010.

BRASIL. Instituto Brasileiro de Geografia e Estatística - IBGE. Educação e trabalho. [Online]. 2013. Disponível em: <http://ibge.gov.br>. Acesso em: 28 abr. 2013.

Ministério do Trabalho e Previdência Social. RAIS - Relação Anual de Informações Sociais. Disponível em: <http://www.rais.gov.br>. Acesso em: 28 abr. 2013.

CAMPOS, A. et al. Atlas da exclusão social no Brasil. São Paulo: Cortez, 2003. 2 v.

DE PAULI, R. C.; NAKABASHI, L.; SAMPAIO, A. V. Mudança estrutural e mercado de trabalho no Brasil. Revista de Economia Política, São Paulo, v.32, n.3, p.459-478, jul./set. 2012.

DIAS, J.; DIAS, M. H.; LIMA, F. F. Crescimento econômico e nível de escolaridade: teoria e estimativas dinâmicas em painel de dados. In: VIII ENCONTRO DE ECONOMIA DA REGIÃO SUL, 2005, Natal. Anais... Natal: Anpec, 2005.

FERNANDES, R.; MENEZES-FILHO, N. A. Escolaridade e demanda relativa por trabalho: uma avaliação para o Brasil nas décadas de 80 e 90. In: CHAHAD, J. P. G.; MENEZES-FILHO, N. A. (Org.). Mercado de trabalho no Brasil: salário, emprego e desemprego numa era de grandes mudanças. São Paulo: LTR, 2002. p.199-221. 1 v. 
LARSON, R.; FARBER, B. Estatística aplicada. 4.ed. São Paulo: Pearson Prentice Hall, 2010.

MENEZES-FILHO, N. A.; RODRIGUES JÚNIOR, M. Tecnologia e demanda por qualificação na indústria brasileira. Revista Brasileira de Economia, Rio de Janeiro, v.75, n.3, p.569-603, 2003.

MINCER, J. Investment in human capital and personal income distribution. Journal of Political Economy, Chicago, v.66, n.4, p.281-302, 1958.

NAKABASHI, L.; FIGUEIREDO, L. Mensurando os impactos diretos e indiretos do capital humano sobre o crescimento. Economia Aplicada, Ribeirão Preto, v.12, n.1, p.151-171, jan./mar. 2008.

VIANA, G.; LIMA, J. F. Capital humano e crescimento econômico. INTERAÇÕES, Campo Grande, v.11, n.2, p.137-148, jul./dez. 2010. 